Title:

Hard Chaos, Quantum Billiards, and Quantum Computers

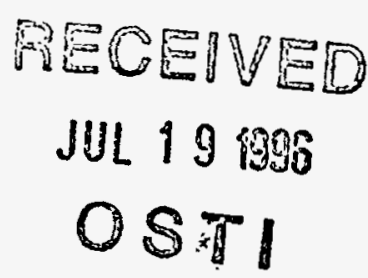

Author(s):

Ronnie Mainieri, T-13

Predrag Cvitanovic (Niels Bohr Institute)

Brosl Hasslacher, T-13

Submitted to:

DOE Office of Scientific and Technical Information (OSTI)

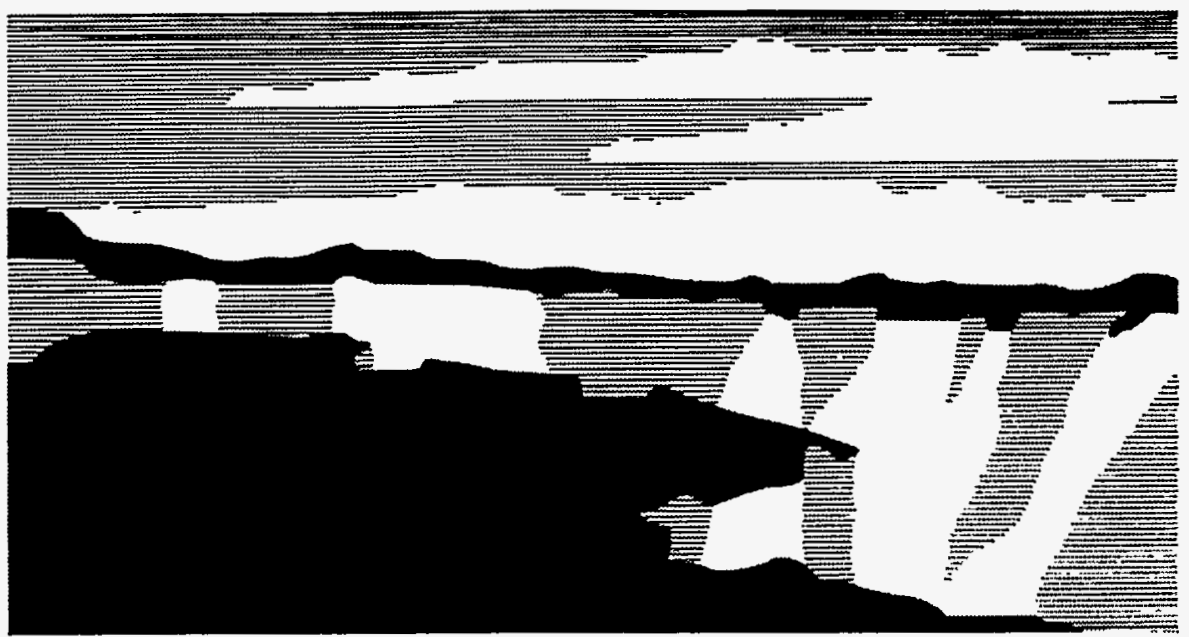

Los Alamos National Laboratory, an affirmative action/equal opportunity employer, is operated by the University of Califomia for the U.S. Department of Energy under contract W-7405-ENG-36. By acceptance of this article, the publisher recognizes that the U.S. Govemment retains a nonexclusive, royaltyfree license to publish or reproduce the published form of this contribution, or to allow others to do so, for U.S. Government purposes. The Los Alamos National Laboratory requests that the publisher identify this article as work performed under the auspices of the U.S. Department of Energy. 


\section{DISCLAIMER}

Portions of this document may be illegible in electronic image products. Images are produced from the best available original document. 


\title{
Hard Chaos, Quantum Billiards, And Quantum Dot Computers
}

Ronnie Mainieri*, Predrag Cvitanovic (Niels Bohr Institute), and Brosl Hasslacher

\begin{abstract}
This is the final report of a three-year, Laboratory-Directed Research and Development (LDRD) project at the Los Alamos National Laboratory (LANL). Research was performed in analytic and computational techniques for dealing with hard chaos, especially the powerful tool of cycle expansions. This work has direct application to the understanding of electrons in nanodevices, such as junctions of quantum wires, or in arrays of dots or antidots. We developed a series of techniques for computing the properties of quantum systems with hard chaos, in particular the flow of electrons through nanodevices. These techniques are providing the insight and tools to design computers with nanoscale components. Recent efforts concentrated on understanding the effects of noise and orbit pruning in chaotic dynamical systems. We showed that most complicated chaotic systems (not just those equivalent to a finite shift) will develop branch points in their cycle expansion. Once the singularity is known to exist, it can be removed with a dramatic increase in the speed of convergence of quantities of physical interest.
\end{abstract}

\section{Background and Research Objectives}

Analytic and computational techniques for dealing with hard chaos, especially the powerful tool of cycle expansions, have direct application to the understanding of electrons in nanodevices such as junctions of quantum wires, or arrays of dots or antidots. The goal of this research project was to develop a set of tools for the design of better nanodevices. We developed techniques for computing the properties of quantum systems with hard chaos, in particular the flow of electrons through nanodevices. These techniques are providing the insight and tools to build computers with nanoscale components.

In systems containing hard chaos, cycle expansions provide a methodology for analytical structural insight, and also the best known computational scheme for evaluating quantities of physical interest [1]. The computation of states of the hydrogen atom in a strong

*Principal investigator, e-mail: ronnie@lanl.gov 
magnetic field $[2,3]$, or the free energy of the Ising model in a random magnetic field $[4,5]$, are examples of problems that are beyond modern supercomputers but that cycle expansions solve in seconds to 15-digit accuracy on ordinary workstations.

In the past few years revolutionary developments have occurred that link two fields, nanolithography [6] and hard chaos [7], that at first seem decoupled. The first of these developments is the descent in scale of the fabrication size of electronic devices that form the heart of computational engines. As we pass the lithographic barrier of 200-nanometer feature size, we encounter a fundamental limit in our ability to construct operational arrays of logic devices. Consequently, a worldwide effort is underway to fabricate and understand devices and their arrays at the 20- to 100-nanometer scale. This has motivated the current interest in few-electron quantum waveguides or "wires," quantum wells or "dots," and the fabrication of large arrays containing these devices, all which will be essential for the design of future generation of massively parallel computers.

As the nanoscale is studied, new physical effects are being discovered that will be the basis for gain and storage devices, but so far the effects have opened a Pandora's box of difficult mathematical physics problems [8-10]. The difficulty lies in the strong coupling between the lithographed potentials and the electrons flowing through them. This leads to scattering states and bound states, which have proven impractical to calculate by large scale computation. Nor have phenomenological approximations been useful, as they miss nonperturbative effects, which are essential in this problem [8].

The second revolution is the ability to calculate physical observables in an extreme regime known as hard chaos, which embraces both classical and quantum domains. The new analytical tool for the revolution is cycle expansions [10]. Hard chaos refers to the Bernoulli (random) character of the flow and is used in contrast to the soft chaos that occurs when the invariant tori of an integrable system are weakly perturbed. Using the KAM theorem and perturbation theory, considerable progress has been made in understanding the soft chaos case, even in the quantum regime [11]. The surprise was that cycle expansions in dynamical systems have led to comparable advances in understanding hard chaos. For example, with the aid of cycle expansions, it has been possible to compute a thermodynamic quantity --- the diffusion constant --- directly from Newton's laws without ever using a probabilistic argument [12]. The quantized version of this problem is exactly the flow of a ballistic electron through an array of antidots. The most spectacular part of the calculation is that it can be done in a few seconds on a workstation-class machine. Other methods for solving the same problem require the use of large supercomputers and do not lead to the same depth of understanding.

Cycle expansions are fundamentally non-perturbative and valid for strong coupling. They are efficient methods for computing with chaotic systems. Two ingredients make cycle 
expansions efficient: First, they work directly in either the infinite volume or the infinite time limit and introduce no finite size or finite time errors. Second, is the phenomenon of the shadowing of longer orbits by shorter ones, allowing errors to decrease at least exponentially fast with the period of the orbits considered.

\section{Importance to LANL's Science and Technology Base and National R\&D Needs}

There is ample evidence that better algorithms have allowed workstation-class computers to solve problems that were once beyond their reach. In recent times, the only exponential problems that have been conquered are those that yield to an heuristic technique (such as simulated annealing or genetic algorithms, to cite two well-known examples), or that have been analyzed in enough detail to generate good enough solutions (for example the routing problem for phone calls). The methods developed in this project are expected to have a similar impact for the Schrodinger equation.

The ray techniques developed in this project can be used in many research areas at the Laboratory. For example, they can be used to improve the computations being carried out in the resonant ultrasound spectroscopy program, to create new non-destructive testing techniques, or to develop better semiconductor simulation techniques.

\section{Scientific Approach and Results}

The research was carried out using a combination of analytical and computational techniques. For this research, it was very important to have access to a workstation with good software development tools. Also, without the opportunity of travel to the Niels Bohr Institute, much of the work carried out under this project would not have been completed. A few of the results obtained under this project are highlighted below.

Exact integrability of chaotic systems. We have developed several models that have nontrivial symbolic dynamics that can be solved exactly. From these exact solutions we have conjectured that generating functionals in chaotic dynamics can be decomposed into two parts. One part is meromorphic only for systems of finite sub-shift and in general has a branch point. The nature of this branch point is completely determined by the topological properties of the system. The other part is determined by an expansion away from constant curvature of the manifold of all geodesics of the system. This part is an entire function. Further work has shown how any dynamical system with strong pruning will develop a cycle expansion with a 
branch point. Knowing the nature of the branch point allows one to remove it and accelerate the convergence of observable quantities.

Pruning. In a cycle expansion, after all the orbits are classified by some symbolic dynamics, one has to determine which orbits to keep to compute the quantities of physical interest. If the right orbits are not kept, the cycle expansion does not converge. This is known as the pruning problem. We added a small amount of noise to a chaotic system and derived the behavior of periodic orbits as a function of the noise strength. This allowed us to pair the orbits according to their sensitivity to noise. This turns out to be the correct way to pair them. With the noise method, one no longer has to be ingenious to determine the pairing; it can be computed through the noise sensitivity. In practice, this has solved the long standing pruning problem.

Exponential work. Suppose we want to determine the amount of computer work to compute the $\mathrm{n}$-th eigenvalue of the Schrodinger equation for a system that is classically chaotic (hyperbolic). If the precision of the eigenvalue is fixed, then the work grows exponentially with $\mathrm{n}$. This surprising result is against all folklore results (which say the work should grow as a power) and it has been carefully checked. This result shows that cycle expansions are computationally more efficient than traditional methods.

Creeping orbits. The geometrical theory of diffraction of Keller has been used to develop a cycle expansion that includes orbits beyond those in the Gutzwiller semiclassical formula. This permits quantum cycle expansions to be used in cases where there are no classical paths.

Noise. The effects of noise model electron-electron collisions, thermal effects, and dissipative and de-coherence effects. We developed a method for incorporating general noise distributions into a chaotic system. The remarkable result is that noise becomes a correction to the action of each periodic orbit of the system. 


\section{References}

[1] R. Artuso, E. Aurell, and P. Cvitanovic, Nonlinearity, 3, 325 (1990).

[2] D. Wintgen, Physical Review Letters, 58, 1589 (1987).

[3] A. Holle, J. Main, G. Wiebusch, and K. H. Welge, Physical Review Letters, 61, 161 (1988).

[4] R. Mainieri, Physical Review Letters, 68, 1965 (1992).

[5] R. Mainieri, Chaos, 2, 91 (1992).

[6] Special issue of Nature on nanophysics (1992).

[7] M. Gutzwiller, Chaos in Classical and Quantum Mechanics, Springer-Verlag, NewYork, 1990.

[8] M. L. Roukes and O. L. Alerhand, Physical Review Letters, 65, 1651 (1990).

[9] P. Cvitanovic, Physica D, 51, 138 (1991).

[10] P. Cvitanovic, Physical Review Letters, 61, 2729 (1988).

[11] H. A. Cerdeira, R. Ramaswamy, M. C. Gutzwiller, and G. Casati, editors, Quantum Chaos, World Scientific, Singapore, 1991.

[12] P. Cvitanovic, P. Gaspard, and T. Schrieber, Chaos, (1992).

\section{DISCLAIMER}

This report was prepared as an account of work sponsored by an agency of the United States Government. Neither the United States Government nor any agency thereof, nor any of their employees, makes any warranty, express or implied, or assumes any legal liability or responsibility for the accuracy, completeness, or usefulness of any information, apparatus, product, or process disclosed, or represents that its use would not infringe privately owned rights. Reference herein to any specific commercial product, process, or service by trade name, trademark, manufacturer, or otherwise does not necessarily constitute or imply its endorsement, recommendation, or favoring by the United States Government or any agency thereof. The views and opinions of authors expressed herein do not necessarily state or reflect those of the United States Government or any agency thereof. 


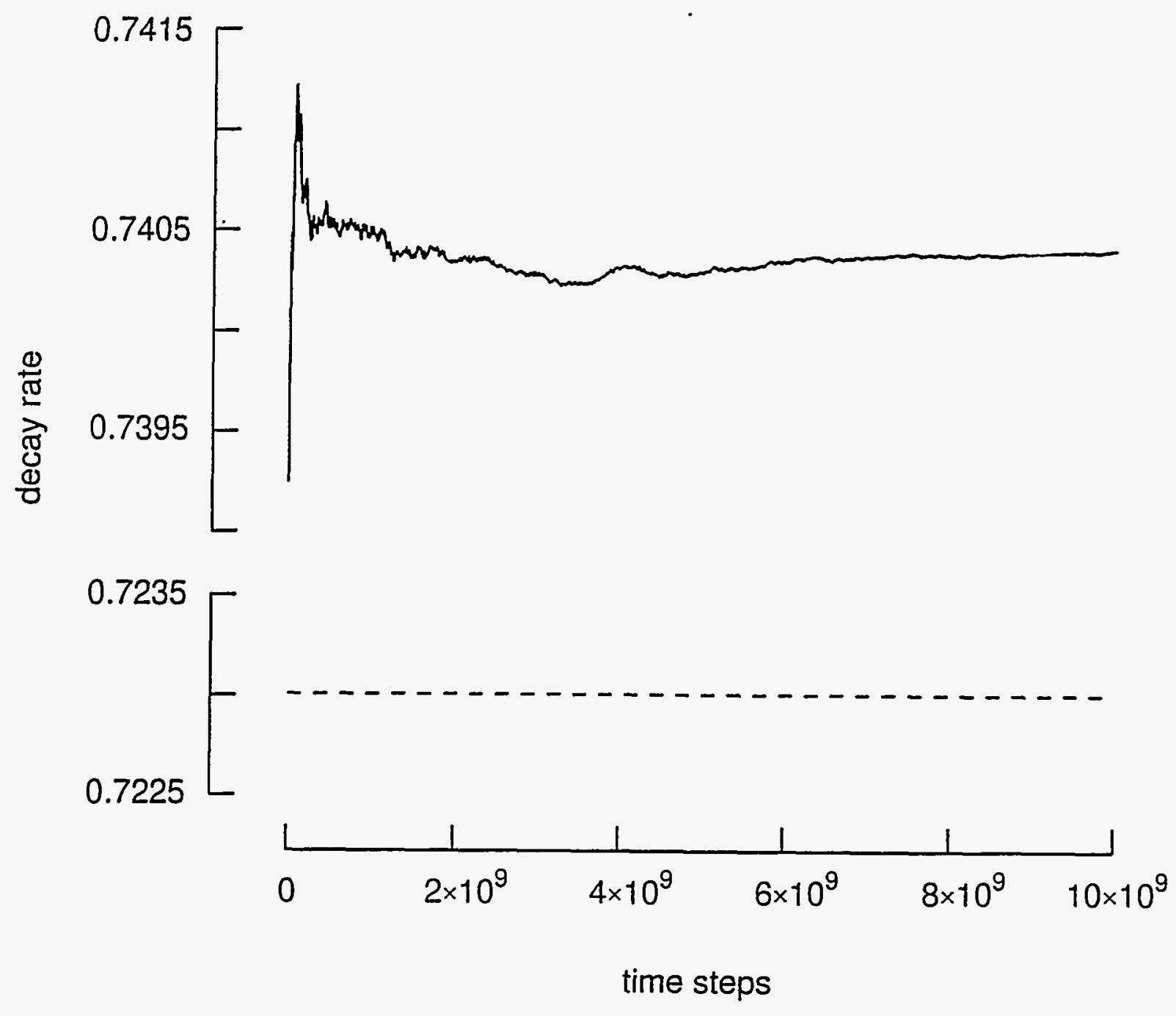

Fig. 1. Monte Carlo simulation (solid line) compared to a cycle expansion (dashed line) of the rate of escape in chaotic scattering. The Monte Carlo simulation has yet to converge to the correct value of 0.7230 , whereas the cycle expansion has converged. 